\title{
Periyodik Eğrilikli İki Komşu İçi Boş Lif İçeren Elastik Ortamdaki Normal Gerilmeler Üzerine
}

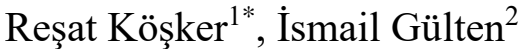 \\ $\mathbf{1}^{*}$ Yıldız Teknik Üniversitesi, Kimya Metalürji Fakültesi, Matematik Mühendisliği Bölümü, İstanbul, Türkiye (ORCID: 0000-0002-0051-340X), kosker@yildiz.edu.tr \\ ${ }^{2}$ Yıldız Teknik Üniversitesi, Fen Bilimleri Enstitüsü, Matematik Mühendisliği Programı, İstanbul, Türkiye (ORCID: 0000-0001-9459-5970), ismailgulten@gmail.com
}

(İlk Geliş Tarihi Aralık 2020 ve Kabul Tarihi Ocak 2021)

(DOI: $10.31590 /$ ejosat.864126)

ATIF/REFERENCE: Köşker, R. \& Gülten, İ. (2021). Periyodik Eğrilikli İki Komşu İçi Boş Lif İçeren Elastik Ortamdaki Normal Gerilmeler Üzerine. Avrupa Bilim ve Teknoloji Dergisi, (22), 316-324.

\section{Öz}

Bu makalede, sonsuz elastik cisme gömülü, sonsuz uzunluklu periyodik eğrilikli birbirine komşu içi boş iki lif olması durumunda lifmatris arayüzeyinde normal gerilmelerin dağlımı incelenmiştir. Komşuluk kavramı, içi boş lifler arasında etkileşim olduğunu ifade etmek için kullanılmıştır. Liflerin orta çizgilerininin aynı düzlemde ve birbirlerine göre aynı fazlı başlangıç eğriliklerine sahip oldukları durum ele alınmıştır. Elastik ortama, lifler yönünde düzgün dağılmış normal kuvvetler etkidiği ve lifler ile matris arasında ideal temas koşullarının sağlandığı düşünülmüş̧ür. Çalışmada, elastisite teorisinin lineerize edilmiş üç boyutlu kesin denklemleri, parçalı-homojen cisim modeli çerçevesinde kullanılmıştır. Böylece, sınır değer probleminin matematiksel modeli kurulabilmiş, bu modelin çözümü için ise sınır formu pertürbasyon yöntemi uygulanmıştır. Buna göre, alan denklemleri ile tamas koşulları, eğilmeyi ifade edecek şekilde tanımlanan küçük parametre cinsinden seri formda yazılarak her bir yaklaşım için, önceki yaklaşımların çözümlerini içeren, ayrı ayrı sınır değer problemleri elde edilmiş ve bu problemler sıfırıncı ve birinci yaklaşımlar için çözülmüştür. Böylece, periyodik eğriliğe sahip içi boş lifler ile matris arayüzeyinde normal gerilmelerle ilgili sayısal sonuçlar elde edilebilmiş ve bu sonuçlar yorumlanmıştır. İçi boş liflerin birbirleri ile etkileşimlerinin, liflerin kalınlık değişimlerinin ve malzeme sabitlerinin, bu gerilme değerlerine etkisi incelenmiş ve tartılmıştır.

\section{On Normal Stresses in Elastic Media Containing Two Neighboring Hollow Fibers with Periodic Curvature}

\begin{abstract}
In this paper, the distribution of normal stresses at the fiber-matrix interface in the case of two neighboring hollow fibers with infinite length periodic curvature embedded in an infinite elastic body is investigated. The concept of neighborhood is used to express the interaction between hollow fibers. The case where the midlines of the fibers are in the same plane and have the same phase initial curvature with respect to each other is considered. It is thought that uniformly distributed normal forces are applied to the elastic medium in the direction of the fibers and ideal contact conditions are provided between the fibers and the matrix. In the study, linearized threedimensional equations of the theory of elasticity is used within the framework of the piecewise-homogeneous body model. Thus, the mathematical model of the boundary value problem is established, and the boundary form perturbation method is applied to the solution of this model. Accordingly, by writing the governing field equations and the complete conditions in series form in terms of the small parameter defined to express the bending, separate boundary value problems containing the solutions of the previous approaches are obtained for each approach. Obtained boundary value problems are solved for the zeroth and first approaches. Thus, numerical results are obtained regarding the normal stresses at the matrix interface with hollow fibers with periodic curvature and these results are
\end{abstract}

* Sorumlu Yazar: kosker@yildiz.edu.tr 
interpreted. The effects of the interaction of hollow fibers with each other, thickness changes of fibers and material constants on the values of these normal stresses are investigated.

Keywords: Two Neighboring Hollow Fibers, Fibrous Composites, Normal Stresses, Periodic Curvature, Composite Materials.

\section{Giriș}

Kompozit malzemeler, en az farklı iki malzemenin mikro veya makro ölçekte bir araya getirilmesiyle oluşturulmuş ve her birinden ayrı ayrı daha üstün özelliklere sahip malzemelerdir. $\mathrm{Bu}$ malzeme, matris adı verilen ana faz ve bunun içine dağılmış takviye elemanlardan oluşur. Takviyenin lif olduğu ve lifli kompozit malzemeler olarak adlandırılan malzemeler, kompozit malzemelerin önemli bir kısmını oluşturmaktadır. Bu malzemelerin, günümüzde, enerji, spor, askeri, otomotiv, denizcilik ve havacılık sektörlerinde, inşaat mühendisliği, biyomedikal uygulamalar ve hatta müzik endüstrisi gibi çok sayıda uygulama alanları mevcuttur. Dolayısıyla, bu malzemelerin mekanik davranışlarının hem teorik hem de deneysel olarak araştırılması önemlidir (Erden ve Ho, 2017).

Lifli kompozit malzemeler üretilirken güçlendirici olarak kullanılan liflerin, farklı geometrik şekillerde malzemeye dahil edilmesiyle farklı özelliklere sahip malzeme elde edilmektedir. Ayrıca, lifler başlangıç eğriliklerine sahip olabilmektedirler. Genellikle, periyodik eğriliğe sahip lifler tasarım gereksinimlerinden oluşturulmakta, yerel eğriliğe sahip lifler ise teknolojik işlemler sonucu ortaya çıkan kusurlar olarak gözlenmektedir (Kelly, 1998; Akbarov ve Guz, 2000; Guz, 2003). Bu malzemeler, dış etkilere maruz kaldıklarında, liflerdeki eğrilik sebebiyle temas yüzeyindeki gerilmeler artar ve bu artış yapışma mukavemetini aşabilir. $\mathrm{Bu}$ durumda, malzemenin mekanik özelliklerini önemli ölçüde etkileyecek makro çatlaklar oluşabilir. Bunlar dışında, liflerin sözü edilen eğrilikleri, stabilite kaybı problemlerinde de kullanılır (Akbarov ve Guz, 2000; Corten ve ark., 1967; Kashtalyan, 2005; Akbarov, 2012). Dolayısıyla, eğrisel yapıya sahip liflerin yer aldığı lifli kompozit malzemelerin teorik olarak çalışılması hem literatüre katkı sağlaması hem de bu malzemelerin yukarıda sayılan alanlarda yapı elemanı olarak kullanılmasından dolayı mühendislik açısından oldukça önemlidir. Bu alandaki teorik çalışmaları, yaklaşık yöntemler kullananlar ve sürekli ortam teorisinin kesin denklemleri çerçevesinde yapılanlar olarak sınıflandırabiliriz (Köşker, 2002; Akbarov ve Guz, 2002). İkinci gruba dahil edebilecek ve üç boyutlu elastisite denklemleri kullanılarak yapılan Akbarov ve Guz (1985) çalışması, periyodik eğrilikli lif yoğunluğunun az olduğu lifli kompozit malzemede lif-matris ara yüzeyindeki gerilme durumları araştırması için bir yöntem önermiştir. Akbarov ve Kosker (2003a, 2003b), Kosker ve Akbarov (2003) çalışmalarında bu yöntem, kompopzit malzemede birbirine yakın periyodik eğrilikli iki lif olması durumuna geliştirilmiş ve liflerin karşılıklı etkileşimlerinin gerilme değerlerine katkısı tartışılmıştır. Kosker ve Ucan (2004), Akbarov ve ark (2004, 2006, 2010, 2016) yayınlarında ise periyodik olarak tek sıra halinde yerleşmiş periyodik eğrilikli liflerin varlığı durumunda gerilme analizleri yapılmıştır. $\mathrm{Bu}$ çalışmalarda parçalı-homojen cisim modeli çerçevesinde elastisite teorisinin lineerize edilmiş üç boyutlu kesin denklemleri kullanılmıştır.

Buraya kadar anılan çalışmalarda, lifler geleneksel malzeme olarak alınmıştır. Karbon nanotüpün pekçok uygulama alanlarında etkin şekilde hayatımıza girmesi ve bu malzemenin, boyutları ile ilgili bazı kısıtlamalar dikkate alındığında, içi boş lif olarak modellenebileceği fikri (Qian ve ark., 2000; Zhuk ve Guz, 2007; Guz ve Dekret, 2008; Maligino ve ark., 2009) yukarıdaki çalışmaların bu alana yayılmasını sağlamıştır. Akbarov (2013) yayınında, çift duvarlı karbon nanotüpün takviye elemanı olarak kullanıldığı malzemede stabilite problemi incelenmiştir. Köşker ve Gülten (2020) çalışmasında periyodik eğrilikli sonsuz uzunluklu içi boș lifin gömülü olduğu kompozit malzeme ele alınmış ve gerilme yayılımı incelenmiştir. Sözü edilen özellikteki lifin malzemede düşük yoğunluklu olduğu durum incelenmiş, dolayısıyla lifler arası etkileşim ihmal edilmiştir. Oysa, uygulamada, genellikle, yapı elemanlarındaki güçlendiriciler, aralarındaki etkileşimin dikkate alınmasını gerektirecek kadar yakındirlar.

$\mathrm{Bu}$ çalışmada, sonsuz bir cisimde, sonsuz uzunlukta periyodik eğrilikli birbirine yakın içi boş iki lif olduğu problem ele alınmış ve bu problemde lif-matris ara yüzeyindeki normal gerilmelerin dağılımı araştırılmıştır. İçi boş liflerin orta çizgilerinin aynı düzlemde ve başlangıç küçük eğriliklerinin aynı fazlı olduğu düşünülmüştür. Elastisite teorisinin üç boyutlu doğrusallaştırılmış denklemleri, parçalı-cisim modeli çerçevesinde kullanılmıştır Ortama, lifler yönünde sonsuzda düzgün dağılmış normal kuvvetler etkidiği durum ele alınmıştır.

\section{Problemin Formülasyonu}

Sonsuz elastik ortama gömülü periyodik eğrilikli içi boş komşu iki lif olduğu, liflerin orta çizgilerinin aynı düzlemde ve başlangıç eğriliklerinin birbirlerine göre aynı fazlı olduğu durum dikkate alınmaktadır (Şekil 1). Liflerin, yüzeylerine dik kesitlerinin iç yarıçaplarının $R$, kalınlıklarının ise $H$ olduğu ve bu değerlerin lifler boyunca değişmediği kabul edilmektedir. Bundan sonra, bu çemberlerin merkezlerinin olduğu eğri, liflerin orta çizgi olarak anılacaktır. Ayrıca, ortama sonsuzda içi boş lifler yönünde $p$ yoğunluklu düzgün dağılmış normal kuvvetlerin etkidiği varsayılmaktadır. Şekil 1'de görüldüğü gibi, merkezleri içi boş liflerin orta çizgilerinin üzerinde bulunan $O_{n} x_{n 1} x_{n 2} x_{n 3}$ kartezyen ve $O_{n} r_{n} \theta_{n} z_{n}$ silindirik koordinat takımları Lagrange koordinatları olarak tanımlanmaktadır. Burada $n=1,2$ olup sırasıyla birinci ve ikinci içi boş lifi göstermektedir. Şekil 1'den de görüldüğü gibi, bu koordinat takınları arasında aşağıdaki bağıntılar vardır.

$x_{12}=x_{22}, x_{13}=x_{23}=z, r_{1} e^{i \theta_{1}}=R_{12}+r_{2} e^{i \theta_{2}}$

İçi boş liflerin orta çizgilerinin $x_{12}=x_{22}=0$ düzleminde olduğu varsayılmakta ve bu çizgilerin denklemleri aşağıdaki gibi alınmaktadır.

$x_{11}=\operatorname{Lsin}\left(\frac{2 \pi}{\ell} x_{13}\right)=\ell \varepsilon \sin \left(\frac{2 \pi}{\ell} x_{13}\right)=\varepsilon \delta_{1}\left(x_{13}\right)$

$x_{21}=\operatorname{Lsin}\left(\frac{2 \pi}{\ell} x_{23}\right)=\ell \varepsilon \sin \left(\frac{2 \pi}{\ell} x_{23}\right)=\varepsilon \delta_{2}\left(x_{23}\right)$

Burada, $L$ içi boş liflerin eğilme genliği ve $\ell$ eğilme periyodu olmak üzere içi boş liflerin başlangıç eğriliklerinin derecesini karakterize eden $\varepsilon=\frac{L}{\ell}$ parametresi tanımlanmıştır $(0<\varepsilon \ll 1)$. 

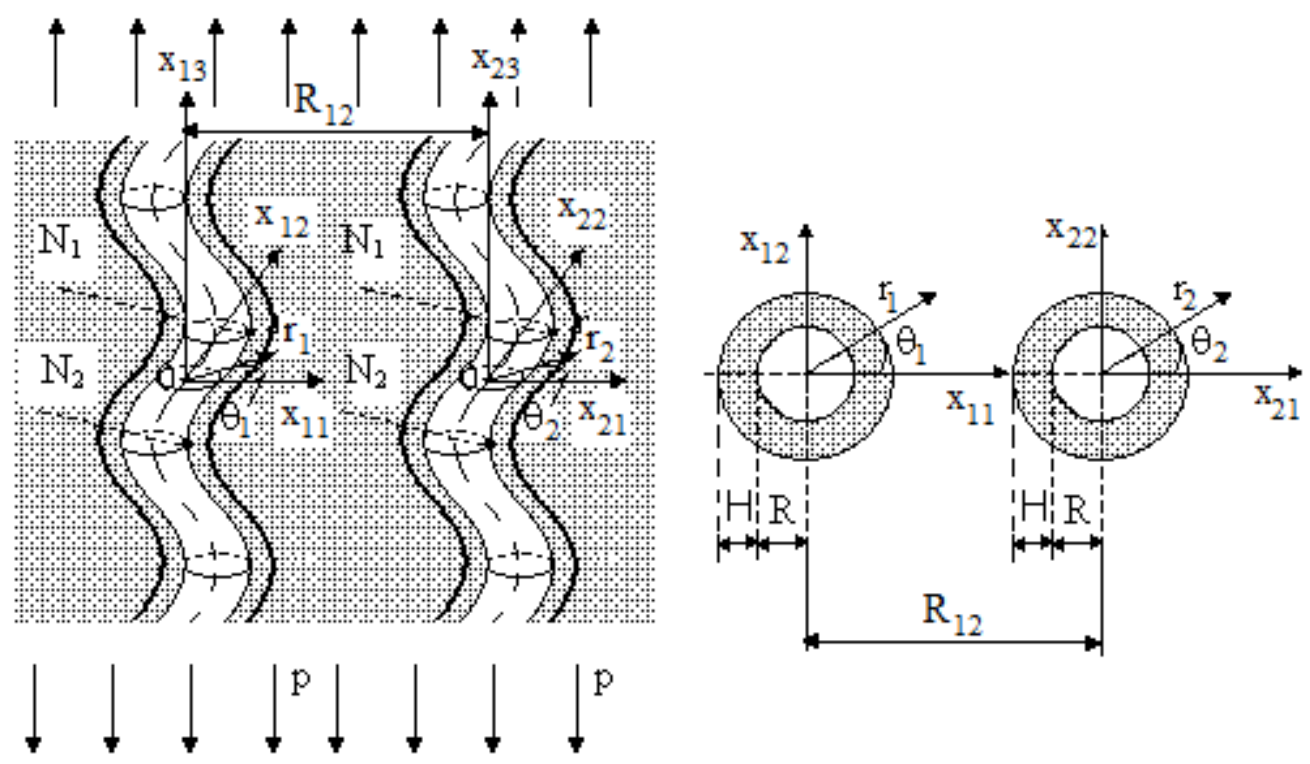

Şekil 1. Problemin geometrisi ve seçilen koordinat takımları

Bundan sonra, sırasıyla, birinci ve ikinci içi boş lifler ile ilgili büyüklükler (21) ve (22) üst indisleri ile, matris ile ilgili büyüklükler ise (1) üst indisi ile gösterilecektir. Sonsuz elastik ortamda ve her bir içi boş lifte sağlanan aşağıdaki alan denklemleri yazılabilir.

$\nabla_{i}\left[\sigma^{(q) i n}\left(g_{n}^{j}+\nabla_{n} u^{(q) j}\right)\right]=0$

$2 \varepsilon_{j m}^{(q)}=\nabla_{j} u_{m}^{(q)}+\nabla_{m} u_{j}^{(q)}+\nabla_{j} u^{(q) n} \nabla_{m} u_{n}^{(q)}$,

$\sigma_{(i n)}^{(q)}=\left(\lambda^{(q)} e^{(q)}\right) \delta_{i}^{n}+2\left(\mu^{(\underline{q})} \varepsilon_{(i n)}^{(q)}\right), e^{(q)}=\varepsilon_{r r}^{(q)}+\varepsilon_{\theta \theta}^{(q)}+\varepsilon_{z z}^{(q)}$

İçi boş liflerin iç yüzeyleri $n=1,2$ olmak üzere $S_{0 n}$, matris ile temas halinde olan dış yüzeyleri ise $S_{1 n}$ ile gösterilsin. İçi boş liflerin dik kesitlerinin iç yarıçaplarının değişmediği varsayımı ile ilgili olan koşul ve lif-matris arasında ideal temas koşulları aşağıdaki gibi ifade edilir.

$\left.\sigma^{(2 q) i n}\left(g_{n}^{j}+\nabla_{n} u^{(2 \underline{q}) j}\right)\right|_{S_{0 q}} n_{\underline{q} j}=0$,

$\left.\sigma^{(2 \underline{q}) i n}\left(g_{n}^{j}+\nabla_{n} u^{(2 \underline{q}) j}\right)\right|_{S_{1 \underline{q}}} n_{q j}=\left.\sigma^{(1) i n}\left(g_{n}^{j}+\nabla_{n} u^{(1) j}\right)\right|_{S_{\underline{1 q}}} n_{\underline{q} j}$,

$\left.u_{j}^{(2 q)}\right|_{S_{1 q}}=\left.u_{j}^{(1)}\right|_{S_{1 \underline{q}}}$

Yukarıda ifade edilen kabullerden aşağıdaki koşullar da yazılır.

$$
\sigma_{z z}^{(1)} \underset{z \rightarrow \infty}{\longrightarrow} p, \quad \sigma_{(i j)}^{(1)} \underset{r_{q} \rightarrow \infty}{\longrightarrow} 0 \quad(i j) \neq(z z)
$$

Verilen formüllerde tansör notasyonu kullanılmıştır ve altı çizili indise göre toplam yoktur.

Böylece, araştırılan problem (3) denklemlerinin (4) ve (5) temas ve sınır koşulları çerçevesinde çözülmesine indirgenmiş olmaktadır. Ulaşılan bu sınır-değer problem, ele alınan problemin matematiksel formülasyonudur.

\section{Sınır-Değer Probleminin Çözümü}

İçi boş liflerin dik kesit koşullarından $\mathrm{S}_{\mathrm{k}}$ yüzeylerinin denklemleri aşağıdaki gibi yazılır.

$$
\begin{aligned}
& r_{q}=\left(1+\varepsilon^{2}\left(\delta_{\underline{q}}^{\prime}\left(t_{3}\right)\right)^{2} \sin ^{2} \theta_{\underline{q}}\right)^{-1}\left\{\left(\varepsilon_{\underline{q}}\left(t_{3}\right)+\varepsilon^{3} \delta_{\underline{q}}\left(t_{3}\right)\left(\delta_{\underline{q}}^{\prime}\left(t_{3}\right)\right)^{2}\right) \sin \theta_{\underline{q}}+\right. \\
& \left.\left[R^{2}-\varepsilon^{2}\left(\delta_{\underline{q}}\left(t_{3}\right)\right)^{2}-\varepsilon^{4}\left(\delta_{\underline{q}}^{\prime}\left(t_{3}\right)\right)^{2}\left(\delta_{\underline{q}}\left(t_{3}\right)\right)^{2}\left(1+\varepsilon^{2}\left(\delta_{\underline{q}}^{\prime}\left(t_{3}\right)\right)^{2}\right) \sin ^{2} \theta_{\underline{q}^{\prime}}\right]^{1 / 2}\right\} \\
& z_{q}=t_{3}-\varepsilon \delta_{\underline{q}}^{\prime}\left(t_{3}\right) r_{\underline{q}}\left(t_{3}\right) \sin \theta_{\underline{q}}+\varepsilon^{2} \delta_{\underline{q}}\left(t_{3}\right) \delta_{\underline{q}}^{\prime}\left(t_{3}\right), \delta_{q}^{\prime}\left(t_{3}\right)=\frac{d \delta_{q}\left(t_{3}\right)}{d t_{3}} \\
& \delta_{q}\left(t_{3}\right)=\ell \sin \left(\frac{2 \pi}{\ell} t_{3}\right)
\end{aligned}
$$

Burada, $t_{3} \in(-\infty,+\infty)$ bir parametredir. (6) denklemleri ve bilinen bazı işlemler kullanılarak, $S_{k}$ yüzeylerinin birim dış normallerinin bileşenleri aşağıdaki gibi bulunur.

$$
\begin{aligned}
& n_{q r}=r_{q}\left(\theta_{\underline{q}}, t_{3}\right) \frac{\partial z_{\underline{q}}\left(\theta_{\underline{q}}, t_{3}\right)}{\partial t_{3}}\left[A_{\underline{q}}\left(\theta_{\underline{q}}, t_{3}\right)\right]^{-1} \\
& n_{q \theta}=\left[\frac{\partial z_{\underline{q}}\left(\theta_{\underline{q}}, t_{3}\right)}{\partial \theta_{\underline{q}}} \frac{\partial r_{\underline{q}}\left(\theta_{\underline{q}}, t_{3}\right)}{\partial t_{3}}-\frac{\partial r_{\underline{q}}\left(\theta_{\underline{q}}, t_{3}\right)}{\partial \theta_{\underline{q}}} \frac{\partial z_{\underline{q}}\left(\theta_{\underline{q}}, t_{3}\right)}{\partial t_{3}}\right]\left[A_{\underline{q}}\left(\theta_{\underline{q}}, t_{3}\right)\right]^{-1} \\
& n_{q z}=-r_{\underline{q}}\left(\theta_{\underline{q}}, t_{3}\right) \frac{\partial r_{\underline{q}}\left(\theta_{\underline{q}}, t_{3}\right)}{\partial t_{3}}\left[A_{\underline{q}}\left(\theta_{\underline{q}}, t_{3}\right)\right]^{-1}
\end{aligned}
$$

(7)'de aşağıdaki notasyon kullanılmıştır. 


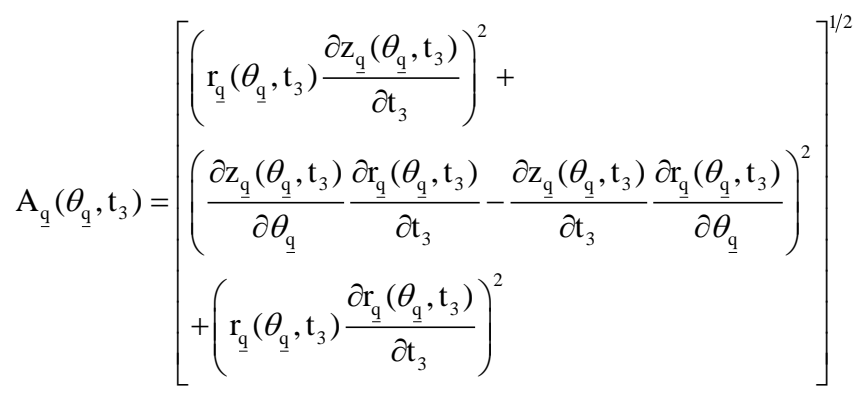

Matematiksel formülasyonu verilen problemin çözümü için Akbarov ve Guz (2000)'de verilen sınır formu pertürbasyon yöntemi kullanılacaktır. Bu yönteme göre aranan tüm büyüklükler daha önce tanımlanan küçük $\varepsilon$ parametresine göre seri formda yazilacaktır.

$$
\left\{\sigma_{(i j)}^{(m)} ; \varepsilon_{(i j)}^{(m)} ; u_{(i)}^{(m)}\right\}=\sum_{q=0}^{\infty} \varepsilon^{q}\left\{\sigma_{(i j)}^{(m), q} ; \varepsilon_{(i j)}^{(m), q} ; u_{(i)}^{(m), q}\right\}
$$

(6) ve (7) ifadeleri $\varepsilon$ cinsinden seri formda aşağıdaki gibi elde edilir.

$r_{q}=R+\sum_{k=1}^{\infty} \varepsilon^{k} a_{\underline{q}}\left(\theta_{\underline{q}}, t_{3}\right), z_{q}=t_{3}+\sum_{k=1}^{\infty} \varepsilon^{k} b_{\underline{q}}\left(\theta_{\underline{q}}, t_{3}\right)$,

$n_{q r}=1+\sum_{k=1}^{\infty} \varepsilon^{k} c_{\underline{q} k}\left(\theta_{\underline{q}}, t_{3}\right), n_{q \theta}=\sum_{k=1}^{\infty} \varepsilon^{k} d_{\underline{q} k}\left(\theta_{\underline{q}}, t_{3}\right)$,

$n_{q z}=\sum_{k=1}^{\infty} \varepsilon^{k} g_{\underline{q} k}\left(\theta_{\underline{q}}, t_{3}\right)$.

Buradaki, $\varepsilon^{k}$ 'nin katsayıları olan $a_{\underline{q} k}\left(\theta_{\underline{q}}, t_{3}\right), \ldots, g_{q k}\left(\theta_{\underline{q}}, t_{3}\right)$ fonksiyonlar (6) ve (7)'den kolaylıkla elde edilebilirler. (3) kullanılarak (9)'daki her bir yaklaşım için sağlanan alan denklemleri bulunur. $n_{q r}, \quad n_{q \theta} \quad n_{q z}$ büyüklüklerinin (10)'daki eşitlikleri kullanılır ve tüm büyüklükler $\left(r_{q}=R+H, \theta_{k}, t_{3}\right)$ ve $\left(r_{q}=R, \theta_{k}, t_{3}\right)$ civarında seriye açılırsa, her bir yaklaşımın temas ve sınır koşulları önceki yaklaşımların büyüklükleri cinsinden elde edilmiş olur.

$\nabla_{n} u^{(q) j, 0}<<1$ olduğundan sıfırıncı yaklaşımla ilgili denklemlerde doğrusal olmayan terimin ihmal edilebildiği durumlar ele alınmıştır. Dolayısıyla, birinci ve sonraki yaklaşımlarda $\left(g_{n}^{j}+\nabla_{n} u^{(q) j, 0}\right)$ terimi $\delta_{n}^{j}$ ile yer değiştirebilir. Ayrıca, sıfırıncı yaklaşımda, $\sigma_{(i j)}^{(q), 0}(i j) \neq z z$ gerilmelerinin $\sigma_{z z}^{(q), 0}$ gerilmelerine göre ihmal edilebilir oldukları varsayılır (Akbarov ve ark, 2004). Bu kabule göre, sıfırıncı yaklaşım için alan denklemleri ile temas koşulları aşağıdaki gibi elde edilmiş olur.

$\nabla_{i} \sigma^{(q) i j, 0}=0, \quad 2 \varepsilon_{i j}^{(q), 0}=\nabla_{j} u_{i}^{(q), 0}+\nabla_{i} u_{j}^{(q), 0}$,

$\sigma_{(i n)}^{(q), 0}=\left(\lambda^{(\underline{q})} e^{(\underline{q}), 0}\right) \delta_{i}^{n}+2\left(\mu^{(q)} \varepsilon_{(i n)}^{(q), 0}\right)$,

$e^{(q), 0}=\varepsilon_{r r}^{(q), 0}+\varepsilon_{\theta \theta}^{(q), 0}+\varepsilon_{z z}^{(q), 0}$

$\left.\sigma_{(i j)}^{(2 q), 0}\right|_{r_{q}=R}=0,\left.\sigma_{(i j)}^{(2 q), 0}\right|_{r_{q}=R+H}=\left.\sigma_{(i j)}^{(1), 0}\right|_{r_{q}=R+H},\left.\quad u_{(i)}^{(2 q), 0}\right|_{r_{q}=R+H}=\left.u_{(i)}^{(1), 0}\right|_{r_{q}=R+H} ;$

$(i j)=r r, r \theta, r z,(i)=r, \theta, z ; q=1,2$

Böylece, sıfırıncı yaklaşım, (11) denklemlerinin (12) temas koşullarıyla çözümüne indirgenmiştir.

e-ISSN: 2148-2683
Birinci yaklaşım için, alan denklemleri aşağıdaki gibi yazılır.

$$
\begin{aligned}
& \nabla_{i}\left[\sigma^{(k) i j, 1}+\sigma^{(k) i n, 0} \nabla_{n} u^{(k) j, 1}\right]=0 \\
& 2 \varepsilon_{i j}^{(k), 1}=\nabla_{j} u_{i}^{(k), 1}+\nabla_{i} u_{j}^{(k), 1} \\
& \sigma_{(i n)}^{(k), 1}=\left(\lambda^{(\underline{k})} e^{(\underline{k}), 1}\right) \delta_{i}^{n}+2\left(\mu^{(\underline{k})} \varepsilon_{(i n)}^{(\underline{k}), 1}\right), \\
& e^{(k), 1}=\varepsilon_{r r}^{(k), 1}+\varepsilon_{\theta \theta}^{(k), 1}+\varepsilon_{z z}^{(k), 1} .
\end{aligned}
$$

Sınır ve temas koşulları ise aşağıdaki gibi bulunur.

$$
\begin{aligned}
{\left[\sigma_{(i) r}\right]^{2 q, 1}+f_{1 \underline{q}}\left[\frac{\partial \sigma_{(i) r}}{\partial r}\right]^{2 \underline{q}, 0}+\phi_{1 \underline{q}}\left[\frac{\partial \sigma_{(i) r}}{\partial z}\right]^{2 \underline{q}, 0}+} \\
\gamma_{r \underline{q}}\left[\sigma_{(i) r}\right]^{2 \underline{q}, 0}+\gamma_{\theta \underline{q}}\left[\sigma_{(i) \theta}\right]^{2 \underline{q}, 0}+\gamma_{z \underline{q}}\left[\sigma_{(i) z}\right]^{2 \underline{q}, 0}=0 \\
{\left[\sigma_{(i) r}\right]_{1,1}^{2 q, 1}+f_{1 \underline{q}}\left[\frac{\partial \sigma_{(i) r}}{\partial r}\right]_{1,0}^{2 \underline{q}, 0}+\phi_{1 \underline{q}}\left[\frac{\partial \sigma_{(i) r}}{\partial z}\right]_{1,0}^{2 \underline{q}, 0}+} \\
\gamma_{r \underline{q}}\left[\sigma_{(i) r}\right]_{1,0}^{2 \underline{q}, 0}+\gamma_{\theta \underline{q}}\left[\sigma_{(i) \theta}\right]_{1,0}^{2 \underline{q}, 0}+\gamma_{z \underline{q}}\left[\sigma_{(i) z}\right]_{1,0}^{2 \underline{q}, 0}=0 \\
{\left[u_{(i)}\right]_{1,1}^{2 q, 1}+f_{1 \underline{q}}\left[\frac{\partial u_{(i)}}{\partial r}\right]_{1,0}^{2 \underline{q}, 0}+\phi_{1 \underline{q}}\left[\frac{\partial u_{(i)}}{\partial z}\right]_{1,0}^{2 \underline{q}, 0}=0 }
\end{aligned}
$$

Burada, aşağıdakiler kullanılmıştır.

$$
\begin{aligned}
& {[\phi]^{2 q, s}=\phi^{(2 q), s},[\phi]_{1, s}^{2 q, s}=\phi^{(2 q), s}-\phi^{(1), s} ; f_{1 q}=\delta_{\underline{q}}\left(t_{3}\right) \cos \theta_{\underline{q}} ;} \\
& \phi_{1 q}=-R \delta_{\underline{q}}^{\prime}\left(t_{3}\right) \cos \theta_{\underline{q}}, \gamma_{r q}=\left(\frac{\delta_{\underline{q}}\left(t_{3}\right)}{R}-\delta_{\underline{q}}^{\prime \prime}\left(t_{3}\right) R\right) \cos \theta_{\underline{q}} ; \\
& \gamma_{\theta q}=-\frac{\delta_{\underline{q}}\left(t_{3}\right)}{R} \sin \theta_{\underline{q}} ; \gamma_{z q}=-\delta_{\underline{q}}^{\prime}\left(t_{3}\right) \cos \theta_{\underline{q}} ; q=1,2
\end{aligned}
$$

Şimdi, sınır-değer problemleri elde edilen sıfırıncı ve birinci yaklaşımların çözümü ile ilgilenilecektir. Kolaylık için, lif malzemelerinin aynı, dolayısıyla bunların Poisson oranlarının eşit $v^{(21)}=v^{(22)}=v^{(2)}\left(v^{(2 q)}\right.$ q. lifin Poisson oran1) ve bunun da matris malzemesinin Poisson oranı olan $v^{(1)}$ değerine eşit olduğu varsayılmıştır elde edilir.

Bu durumda, sıfırıncı yaklaşımın çözümü aşağıdaki gibi $\sigma_{z z}^{(1), 0}=p ; \sigma_{z z}^{(21), 0}=\sigma_{z z}^{(22), 0}=\frac{E^{(2)}}{E^{(1)}} p ; \quad \varepsilon_{z z}^{(21), 0}=\varepsilon_{z z}^{(22), 0}=\varepsilon_{z z}^{(1), 0}=\frac{p}{E^{(1)}} ;$ $z=z_{1}=z_{2}, \quad u_{z}^{(21), 0}=u_{z}^{(22), 0}=u_{z}^{(1), 0}=\varepsilon_{z z}^{(1), 0} z ; \quad \sigma_{(i j)}^{(2 q), 0}=\sigma_{(i j)}^{(1), 0}=0 ;$ $(i j)=r r, \theta \theta, r \theta, \theta z, r z$

(18)'deki $E^{(1)}, E^{(2)}$ sirasıyla, matris ve lif malzemelerinin elastisite sabitleridirler.

Sıfırıncı yaklaşımın çözümü olarak elde edilen (18) ifadeleri birinci yaklaşımın denge denklemleri olan (13)'de kullanılırsa aşağıdakiler elde edilir.

$$
\begin{aligned}
& \frac{\partial \sigma_{r r}^{(q), 1}}{\partial r_{\underline{q}}}+\frac{1}{r_{\underline{q}}} \frac{\partial \sigma_{r \bar{\theta}}^{(q), 1}}{\partial \theta_{\underline{q}}}+\frac{\partial \sigma_{r z}^{(q), 1}}{\partial z_{\underline{q}}}+\frac{1}{r_{\underline{q}}}\left(\sigma_{r r}^{(\underline{q}), 1}-\sigma_{\theta \bar{\theta}}^{(\underline{q}), 1}\right)=0, \\
& \frac{\partial \sigma_{r \bar{\theta}}^{(\underline{q}), 1}}{\partial r_{\underline{q}}}+\frac{1}{r_{\underline{q}}} \frac{\partial \sigma_{\theta \bar{\theta}}^{(\underline{q}), 1}}{\partial \theta_{\underline{q}}}+\frac{\partial \sigma_{\theta z}^{(q), 1}}{\partial z_{\underline{q}}}+\frac{2}{r_{\underline{q}}} \sigma_{r \bar{\theta}}^{(q), 1}=0,
\end{aligned}
$$


$\frac{\partial \sigma_{r z}^{(q), 1}}{\partial r_{q}}+\frac{1}{r_{q}} \frac{\partial \sigma_{\theta z}^{(q), 1}}{\partial \theta_{q}}+\frac{\partial \sigma_{z z}^{(q), 1}}{\partial z_{q}}+\frac{1}{r_{q}} \sigma_{r z}^{(q), 1}=0$

$\mathrm{Bu}$ denklemler, elastisite teorisinin üç boyutlu doğrusallaşmış denklemleri ile çakışırlar. Benzer şekilde, (14) iafadeleri aşağıdakilere dönüşür.

$$
\begin{aligned}
& \varepsilon_{r r}^{(q), 1}=\frac{\partial u_{r}^{(\underline{q}), 1}}{\partial r_{\underline{q}}}, \varepsilon_{r \theta}^{(q), 1}=\frac{1}{2}\left(\frac{1}{r_{\underline{q}}} \frac{\partial u_{r}^{(\underline{q}), 1}}{\partial \theta_{\underline{q}}}+\frac{\partial u_{\theta}^{(\underline{q}), 1}}{\partial r_{\underline{q}}}-\frac{u_{\theta}^{(\underline{q}), 1}}{r_{\underline{q}}}\right), \\
& \varepsilon_{r z}^{(q), 1}=\frac{1}{2}\left(\frac{\partial u_{z}^{(q), 1}}{\partial r_{\underline{q}}}+\frac{\partial u_{r}^{(\underline{q}), 1}}{\partial z_{\underline{q}}}\right), \varepsilon_{\theta \theta}^{(q), 1}=\frac{1}{r_{\underline{q}}} \frac{\partial u_{\theta}^{(\underline{q}), 1}}{\partial \theta_{\underline{q}}}+\frac{u_{r}^{(\underline{q}), 1}}{r_{\underline{q}}}, \\
& \varepsilon_{\theta z}^{(q), 1}=\frac{1}{2}\left(\frac{\partial u_{\theta}^{(\underline{q}), 1}}{\partial z_{\underline{q}}}+\frac{1}{r_{\underline{q}}} \frac{\partial u_{z}^{(\underline{q}), 1}}{\partial \theta_{\underline{q}}}\right) \quad \varepsilon_{z z}^{(q), 1}=\frac{\partial u_{z}^{(q), 1}}{\partial z_{\underline{q}}},
\end{aligned}
$$

Sıfırıncı yaklaşımın çözümleri dikkate alınırsa, birinci yaklaşıma ait ve (16) ile verilen temas ve sınır koşulları aşağıdaki hale gelirler.

$\left[\sigma_{r r}\right]^{2 q, 1}=0,\left[\sigma_{r \theta}\right]^{2 q, 1}=0,\left[\sigma_{r z}\right]^{2 q, 1}=\delta_{\underline{q}}^{\prime}\left(t_{3}\right) \sigma_{z z}^{(2), 0} \cos \theta_{\underline{q}}$,

$\left[\sigma_{r r}\right]_{1,1}^{2 q, 1}=0,\left[\sigma_{r \theta}\right]_{1,1}^{2 q, 1}=0$,

$\left[\sigma_{r z}\right]_{1,1}^{2 q, 1}=\delta_{\underline{q}}^{\prime}\left(t_{3}\right)\left(\sigma_{z z}^{(1), 0}-\sigma_{z z}^{(2), 0}\right) \cos \theta_{\underline{q}}$,

$\left[u_{r}\right]_{1,1}^{2 q, 1}=0,\left[u_{\theta}\right]_{1,1}^{2 q, 1}=0,\left[u_{z}\right]_{1,1}^{2 q, 1}=0$

Bu denklemlerin çözümü için, (19) denklemlerini de dikkate alan Akbarov ve Guz (2000)'de verilen aşağıdaki gösterilim kullanılır.

$u_{r}^{(q), 1}=\frac{1}{r_{q}} \frac{\partial}{\partial \theta_{q}} \psi^{(\underline{q})}-\frac{\partial^{2}}{\partial r_{q} \partial z} \chi^{(\underline{q})} ;$

$u_{\theta}^{(q), 1}=-\frac{\partial}{\partial r_{\underline{q}}} \psi^{(\underline{q})}-\frac{1}{r_{q}} \frac{\partial^{2}}{\partial \theta_{\underline{q}} \partial z} \chi^{(\underline{q})} ; \Delta_{1}^{(q)}=\frac{\partial^{2}}{\partial r_{q}^{2}}+\frac{1}{r_{\underline{q}}} \frac{\partial}{\partial r_{\underline{q}}}+\frac{1}{r_{\underline{q}}^{2}} \frac{\partial^{2}}{\partial \theta_{\underline{q}}^{2}}$

$u_{z}^{(q), 1}=\left(\lambda^{(\underline{q})}+\mu^{(\underline{q})}\right)^{-1}\left(\begin{array}{l}\left(\lambda^{(\underline{q})}+2 \mu^{(\underline{q})}\right) \Delta_{1}^{(q)}+ \\ \left(\mu^{(q)}+\sigma_{z z}^{(q), 0}\right) \frac{\partial^{2}}{\partial z^{2}}\end{array}\right) \chi^{(\underline{q})}$

Buradaki $\quad \psi^{(q)}, \chi^{(q)}$ fonksiyonları aşağıdaki diferansiyel denklemleri sağlarlar.

$$
\begin{aligned}
& \left(\Delta_{1}^{(\underline{q})}+\left(\xi_{1}^{(q)}\right)^{2} \frac{\partial^{2}}{\partial z^{2}}\right) \psi^{(\underline{q})}=0 ; \\
& \left(\Delta_{1}^{(\underline{q})}+\left(\xi_{2}^{(q)}\right)^{2} \frac{\partial^{2}}{\partial z^{2}}\right)\left(\Delta_{1}^{(q)}+\left(\xi_{3}^{(q)}\right)^{2} \frac{\partial^{2}}{\partial z^{2}}\right) \chi^{(\underline{q})}=0
\end{aligned}
$$

$\xi_{i}^{(q)}(q=21,22,1 ; i=1,2,3)$ 'ler sabitlerdir (Kosker ve Akbarov, 2003). (23) diferansiyel denklemlerinin, (21)'deki koşulların sağ tarafı da dikkate alınan çözümleri aşağıdaki gibi elde edilir.

$\psi^{(2 q), 1}=\alpha \sin \alpha z \sum_{n=-\infty}^{\infty}\left\{\begin{array}{l}C_{n}^{(2 q)} I_{n}\left(\xi_{1}^{(2 q)} \alpha r_{\underline{q}}\right)+ \\ D_{n}^{(2 q)} K_{n}\left(\xi_{1}^{(2 \underline{q})} \alpha r_{\underline{q}}\right)\end{array}\right\} \exp \left(i n \theta_{\underline{q}}\right)$,

$$
\chi^{(2 q), 1}=\cos \alpha z \sum_{n=-\infty}^{\infty}\left\{\begin{array}{l}
A_{n}^{(2 q)} I_{n}\left(\xi_{2}^{(2 q)} \alpha r_{\underline{q}}\right)+ \\
B_{n}^{(2 q)} I_{n}\left(\xi_{3}^{(2 q)} \alpha r_{\underline{q}}\right)+ \\
E_{n}^{(2 q)} K_{n}\left(\xi_{2}^{(2 \underline{q})} \alpha r_{\underline{q}}\right)+ \\
F_{n}^{(2 q)} K_{n}\left(\xi_{3}^{(2 \underline{q})} \alpha r_{\underline{q}}\right)
\end{array}\right\} \exp \left(i n \theta_{\underline{q}}\right),
$$

$$
\psi^{(1), 1}=\alpha \sin \alpha z \sum_{q=1}^{2} \sum_{n=-\infty}^{\infty} C_{n}^{(1) q} K_{n}\left(\xi_{1}^{(1)} \alpha r_{q}\right) \exp \left(i n \theta_{q}\right),
$$$$
\chi^{(1), 1}=\cos \alpha z \sum_{q=1}^{2} \sum_{n=-\infty}^{\infty}\left[\begin{array}{l}
A_{n}^{(1) q} K_{n}\left(\xi_{2}^{(1)} \alpha r_{q}\right)+ \\
B_{n}^{(1) q} K_{n}\left(\xi_{3}^{(1)} \alpha r_{q}\right)
\end{array}\right] \exp \left(i n \theta_{q}\right)
$$

(24) ve (25), $\alpha=2 \pi / \ell$ ve $I_{n}(x), K_{n}(x)$ fonksiyonları ise, sırasıyla, sanal argümanlı Bessel fonksiyonları ve Macdonald fonksiyonlarıdır. $\quad A_{n}^{(2 q)}, \ldots, C_{n}^{(2 q)}, \quad A_{n}^{(1) q}, \ldots, C_{n}^{(1) q}$ bilinmeyenleri kompleks sabitlerdir ve aşağıdaki ilişkileri sağlarlar.

$A_{n}^{(2 q)}=\overline{A_{-n}^{(2 q)}}, B_{n}^{(2 q)}=\overline{B_{-n}^{(2 q)}}, \quad C_{n}^{(2 q)}=\overline{C_{-n}^{(2 q)}}$,

$\operatorname{Im} A_{0}^{(2 q)}=\operatorname{Im} B_{0}^{(2 q)}=\operatorname{Im} C_{0}^{(2 q)}=0$

$A_{n}^{(1) q}=\overline{A_{-n}^{(1) q}}, B_{n}^{(1) q}=\overline{B_{-n}^{(1) q}}, \quad C_{n}^{(1) q}=\overline{C_{-n}^{(1) q}}$,

$\operatorname{Im} A_{0}^{(1) q}=\operatorname{Im} B_{0}^{(1) q}=\operatorname{Im} C_{0}^{(1) q}=0$

$\left(r_{2}, \theta_{2}\right)$ koordinatlarındaki büyüklükleri $\left(r_{1}, \theta_{1}\right)$ koordinatları cinsinden veya tersini yapmak için toplam teoreminden (Watson, 1966) ve koordinatlar arasındaki ilişkilerden yararlanılır.

$$
\begin{aligned}
& r_{\underline{\underline{m}}} \exp i \theta_{\underline{\underline{m}}}=R_{\underline{\underline{m}} \underline{\underline{n}}} \exp i \phi_{\underline{\underline{\underline{m}}} \underline{\underline{n}}}+r_{\underline{\underline{n}}} \exp i \theta_{\underline{\underline{n}}}, \\
& K_{v}\left(c r_{\underline{\underline{r}}}\right) \exp i v \theta_{\underline{\underline{n}}}=\sum_{k=-\infty}^{\infty}\left[\begin{array}{l}
(-1)^{v} I_{k}\left(c r_{\underline{\underline{m}}}\right) K_{v-k}\left(c R_{\underline{\underline{m}} \underline{\underline{n}}}\right) . \\
\exp \left[i(v-k) \phi_{\underline{\underline{m}} \underline{\underline{n}}}\right] \exp i k \theta_{\underline{\underline{m}}}
\end{array}\right], m n=12 ; 21 \\
& m ; n=1,2 ; r_{m}<R_{m n} ; R_{12}=R_{21} ; \phi_{12}=0 ; \phi_{21}=\pi .
\end{aligned}
$$

Bütün bunlar, (21)'de kullanıllı ve problemin geometrisinden kaynaklanan simetri de dikkate alınırsa sonsuz boyutlu denklemler sistemine ulaşılır. Böylece, yakınsaklık kriteri kullanılarak yeteri kadar denklem alınıp birinci yaklaşıma ait bilinmeyenler belirlenerek istenen normal gerilme değerleri hesaplanabilir.

\section{Sayısal Sonuçlar ve Değerlendirme}

Sirasılyla, teğet $\tau$ ve binormal e vektörleri yönündeki normal gerilmeler olan $\sigma_{\tau \tau}$ ve $\sigma_{e e}$ gerilmelerinin, içi boş lif ile matris ara yüzeyi üzerinde matrise ait değerleri ile ilgilenilecektir. Eğer içi boş liflerin eğriliği ihmal edilirse, yani $\varepsilon=0$ alınırsa, $\sigma_{\tau \tau}^{(1)}=\sigma_{z z}^{(1), 0}+\varepsilon \sigma_{z z}^{(1), 1}+O\left(\varepsilon^{2}\right) \quad$ ve $\quad \sigma_{e e}^{(1)}=\varepsilon \sigma_{\theta \theta}^{(1), 1}+O\left(\varepsilon^{2}\right)$ olduğundan gerilmeler sirasıyla $\sigma_{\tau \tau}^{(1)}=\sigma_{z z}^{(1), 0}$ ve $\sigma_{e e}^{(1)}=0$ olur.

Sayısal sonuçlar sıfırıncı ve birinci yaklaşımlar çerçevesinde elde edilmiştir. Bu hesaplar, gerilme değerlerinin parametrelere göre davranışını görmek için yeterlidir. Sonraki yaklaşımlar sayısal değerleri sadece nicelik olarak biraz daha iyileştirebilir (Akbarov ve Guz, 2000). Her iki normal gerilme, mutlak değerce maksimum değerlerini aldıkları $\theta=0, \alpha t_{3}=\pi / 2$ noktasında hesaplanmıştır. Ayrıca, Poisson oranları $v^{(1)}=v^{(21)}=v^{(22)}=0.3$ olarak kullanılmıştır. Gerilme değerlerine, lifler arasındaki uzaklığın etkisini görmek için 
$\rho=R_{12} / R$, elastisite modulleri oranının etkisini görmek için $E=E^{(2)} / E^{(1)}$, lif yarıçap değişiminin etkisini görmek için $\gamma_{1}=2 \pi(R+H) / \ell=\alpha(R+H)$ ve içi boş lifin kalınlığının etkisini

$$
\sigma_{\tau \tau} / \mathrm{p}
$$

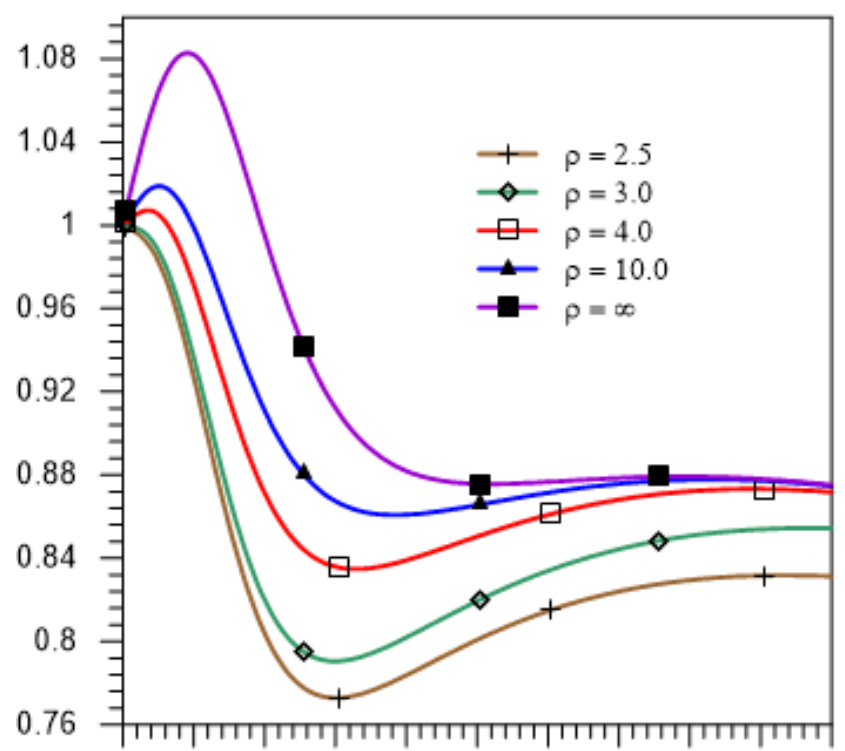

$\begin{array}{lllllllllll}0 & 0.2 & 0.4 & 0.6 & 0.8 & 1 & 1.2 & 1.4 & 1.6 & 1.8 & 2\end{array}$

(a) görmek için $\quad \gamma_{2}=H /(R+H)$ boyutsuz parametreleri tanımlanmıştır.

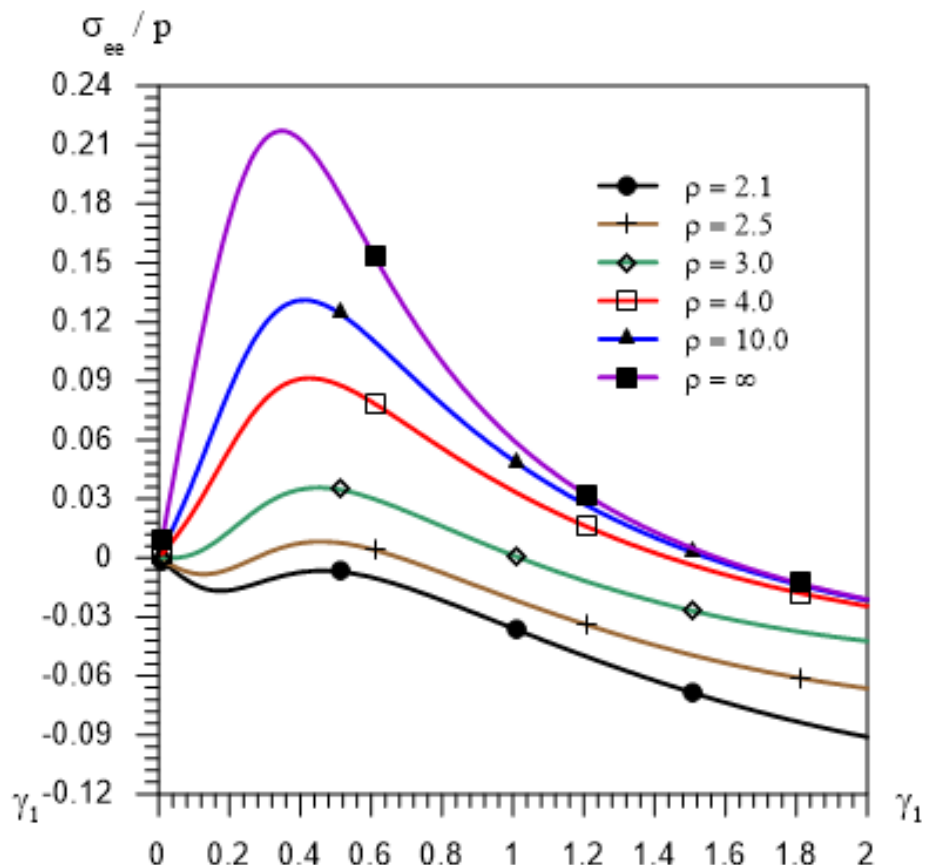

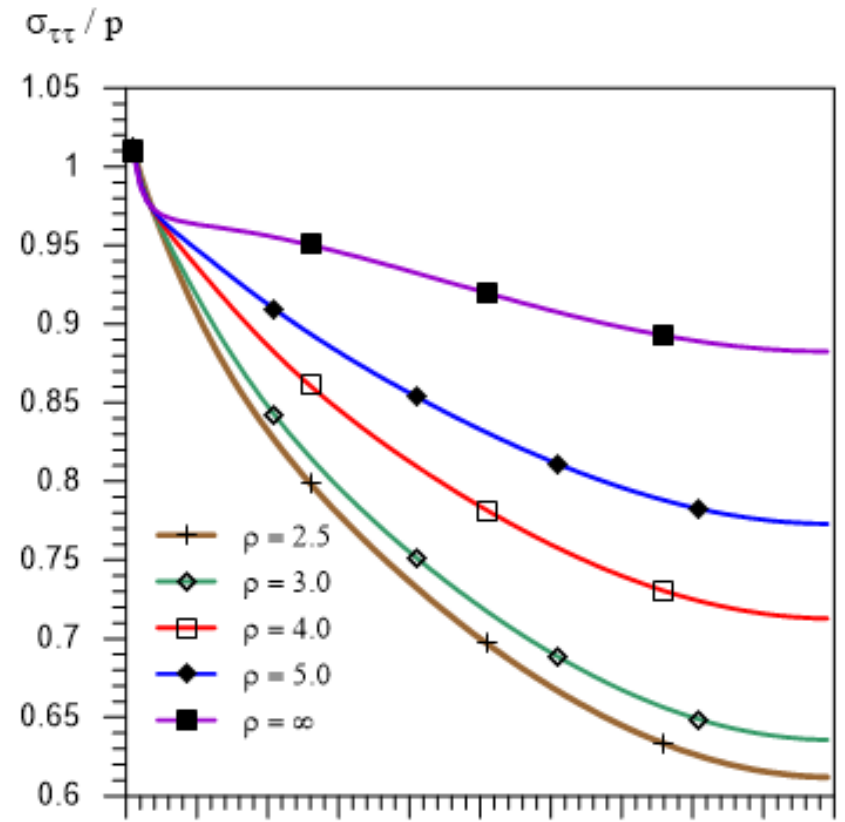

$\begin{array}{lllllllllll}0 & 0.1 & 0.2 & 0.3 & 0.4 & 0.5 & 0.6 & 0.7 & 0.8 & 0.9 & 1\end{array}$

(a)

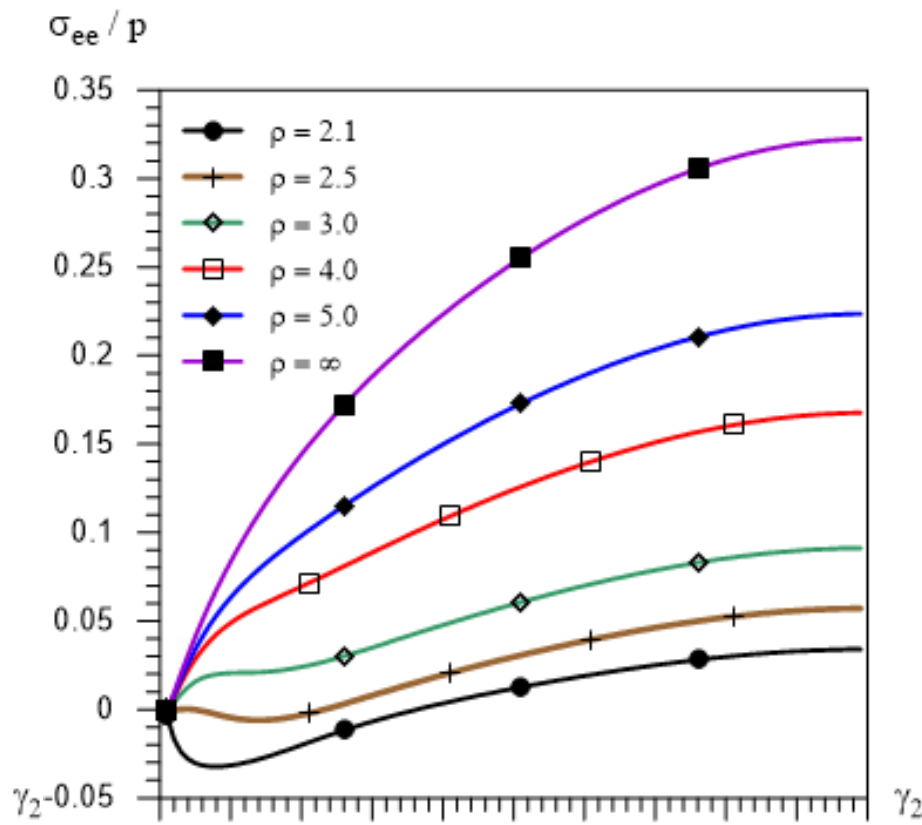

$\begin{array}{lllllllllll}0 & 0.1 & 0.2 & 0.3 & 0.4 & 0.5 & 0.6 & 0.7 & 0.8 & 0.9 & 1\end{array}$

(b)

Şekil 3. Çeşitli $\rho$ değerlerinde $\sigma_{\tau \tau} / p$ (a) ve $\sigma_{e e} / p$ (b) dĕgerlerinin $\gamma_{2}$ parametresi ile değişimi $\left(E=100, \gamma_{2}=0.5, \varepsilon=0.015\right)$ 
Şekil 2 ve 3'de çeşitli $\rho$ değerlerinde $\sigma_{\tau \tau} / p$ (a) ve $\sigma_{e e} / p$ (b) normal gerilme değerlerinin sirası $\gamma_{1}$ ve $\gamma_{2}$ parametrelerine göre değişimi görülmektedir. Her iki grafikte $E=100, \varepsilon=0.015$ değerleri kullanılmış, Şekil 2'de $\gamma_{2}=0.3$, Şekil 3'de ise $\gamma_{1}=0.5$ alınmıştır. Bu grafiklerden, hesaplanan normal gerilme değerlerinin lif yarıçapı ile ilişkisinin monoton olmadığı, oysa $\sigma_{\tau \tau} / p$ 'nin lif kalınlığı ile değişimi monoton iken $\sigma_{e e} / p$ 'nin lif kalınlığı ile değişiminin, lifler birbirine yaklaştıkça, monoton olmadı̆̆ı söylenebilir. Diğer taraftan, $\sigma_{\tau \tau} / p$ normal gerilme değerleri lifler birbirine yaklaştıkça azalmakta, ancak $\sigma_{e e} / p$ normal gerilme değerleri ise lif yarıçapının belli bir değerine kadar (yaklaşık $\gamma_{1}=1$ ), lifler birbirine yaklaşırken azalmakta iken lif yarıçapının bu değerinden büyük değerleri için ise lifler birbirine yaklaştıkça artmaktadır. Bu grafiklerde $\rho=\infty$ durumu, içi boş lifler arası etkileşimin olmadığı Köşker ve Gülten (2020) çalışmasındaki probleme karşı gelen değerleri vermektedir.

Tablo 1. $\gamma_{1}=0.5, \gamma_{2}=0.3$ ve çeşitli $E, \rho$ ve $\varepsilon$ değerlerinde normal gerilmeler

\begin{tabular}{|c|c|c|c|c|}
\hline$E$ & $\rho$ & $\varepsilon$ & $\frac{\sigma_{\tau \tau}}{p}$ & $\frac{\sigma_{e e}}{p}$ \\
\hline \multirow{9}{*}{10} & \multirow{3}{*}{2.5} & 0.01 & 0,94944 & 0,00040 \\
\hline & & 0.015 & 0,92416 & 0,00061 \\
\hline & & 0.02 & 0,89889 & 0,00081 \\
\hline & \multirow{3}{*}{3.0} & 0.01 & 0,95467 & 0,01190 \\
\hline & & 0.015 & 0,93201 & 0,01785 \\
\hline & & 0.02 & 0,90934 & 0,02380 \\
\hline & \multirow{3}{*}{4.0} & 0.01 & 0,95889 & 0,01852 \\
\hline & & 0.015 & 0,93834 & 0,02778 \\
\hline & & 0.02 & 0,91778 & 0,03704 \\
\hline \multirow{9}{*}{50} & \multirow{3}{*}{2.5} & 0.01 & 0,90610 & 0,01119 \\
\hline & & 0.015 & 0,85915 & 0,01679 \\
\hline & & 0.02 & 0,81220 & 0,02238 \\
\hline & \multirow{3}{*}{3.0} & 0.01 & 0,92510 & 0,03494 \\
\hline & & 0.015 & 0,88765 & 0,05241 \\
\hline & & 0.02 & 0,85021 & 0,06988 \\
\hline & \multirow{3}{*}{4.0} & 0.01 & 0,93906 & 0,05028 \\
\hline & & 0.015 & 0,90859 & 0,07542 \\
\hline & & 0.02 & 0,87812 & 0,10056 \\
\hline
\end{tabular}

\begin{tabular}{|c|c|c|c|c|}
\hline$E$ & $\rho$ & $\varepsilon$ & $\frac{\sigma_{\tau \tau}}{p}$ & $\frac{\sigma_{e e}}{p}$ \\
\hline \multirow{9}{*}{100} & \multirow{3}{*}{2.5} & 0.01 & 0,86417 & 0,02335 \\
\hline & & 0.015 & 0,79626 & 0,03502 \\
\hline & & 0.02 & 0,72835 & 0,04670 \\
\hline & \multirow{3}{*}{3.0} & 0.01 & 0,89718 & 0,05913 \\
\hline & & 0.015 & 0,84577 & 0,08869 \\
\hline & & 0.02 & 0,79436 & 0,11826 \\
\hline & \multirow{3}{*}{4.0} & 0.01 & 0,92154 & 0,08374 \\
\hline & & 0.015 & 0,88231 & 0,12561 \\
\hline & & 0.02 & 0,84308 & 0,16748 \\
\hline \multirow{9}{*}{150} & \multirow{3}{*}{2.5} & 0.01 & 0,83903 & 0,03104 \\
\hline & & 0.015 & 0,75855 & 0,04656 \\
\hline & & 0.02 & 0,67807 & 0,06209 \\
\hline & \multirow{3}{*}{3.0} & 0.01 & 0,88032 & 0,07409 \\
\hline & & 0.015 & 0,82049 & 0,11114 \\
\hline & & 0.02 & 0,76065 & 0,14819 \\
\hline & \multirow{3}{*}{4.0} & 0.01 & 0,91105 & 0,10456 \\
\hline & & 0.015 & 0,86658 & 0,15684 \\
\hline & & 0.02 & 0,82211 & 0,20912 \\
\hline
\end{tabular}

Tablo 2. Denklem sayısı ile gerilme değerlerinin yakınsaması ( $E=100, \gamma_{1}=0.5 \rho=3.0, \gamma_{2}=0.3$ )

\begin{tabular}{|c|c|c|c|c|c|c|c|}
\hline & \multicolumn{7}{|c|}{ Denklem Say1s1 } \\
\cline { 2 - 8 } & 42 & 51 & 60 & 69 & 78 & 87 & 186 \\
\hline$\sigma_{\tau \tau} / \mathrm{p}$ & 0.7959 & 0.7959 & 0.7959 & 0.7962 & 0.7962 & 0.7962 & 0.7962 \\
\hline$\sigma_{\text {ее }} / \mathrm{p}$ & 0.0347 & 0.0347 & 0.0347 & 0.0349 & 0.0349 & 0.0349 & 0.0350 \\
\hline
\end{tabular}


Tablo $1 \gamma_{1}=0.5, \gamma_{2}=0.3$ için çeşitli $E, \rho$ ve $\varepsilon$ değerlerinde normal gerilmeleri göstermektedir. $\mathrm{Bu}$ tablodaki verilerden, eğilmenin derecesi $(\varepsilon)$ ve elastisite modülü oranı $(E)$ arttıkça $\sigma_{\tau \tau} / p$ değerleri azalırken $\sigma_{e e} / p$ değerlerinin arttığ gözlenmektedir. Ayrıca, liflerin birbirine yaklaşmasının söz konusu normal gerilmeleri azalttığı da söylenebilir.

Tablo 2'de, birinci yaklaşımla ilgili bilinmeyenleri elde etmek için elde edilen sonsuz denklemler sisteminin sonlu ile değiştirilebileceğini veren sayısal değerlerin yakınsaklığı görülmektedir. Burada, gerilme değerleri $E=100, \gamma_{1}=0.5$ $\rho=3.0, \gamma_{2}=0.3$ parametre değerlerinde hesaplanmıştır.

Sayısal verilere, FTN77 programlama dilinde kodlama yapılarak ulaşılmıştır. Liflerin kalınlıklarının artırılması ve lifler arasındaki uzaklığın büyük alınması durumunda elde edilen sayısal veriler, Köşker ve Uçan (2004) çalışmasında yer alan ve periyodik eğrilikli içi dolu lif problemindeki normal gerilmelerle çakışmakta, bu ise kullanılan algoritmanın ve yapılan hesapların doğruluğunu göstermektedir.

\section{Sonuç}

$\mathrm{Bu}$ çalışmada, elastisite teorisinin üç boyutlu dağrusallaştırılmış denklemleri kullanılarak parçalı-homojen cisim modeli çerçevesinde sonsuz uzunluklu periyodik başlangıç eğriliğine sahip içi boş komşu iki lifin gömülü olduğu sonsuz elastik ortamda normal gerilmeler çalışılmıştır. Liflerin orta çizgilerinin aynı düzlem üzerinde ve başlangıç eğriliklerinin birbirlerine göre aynı fazlı olduğu düşünülmüştür. Ayrıca, lifler yönünde sonsuzda düzgün dağılmış normal kuvvetlerin varlığ dikkate alınmıştır. Liflerin kalınlıklarının ve yarıçaplarının sabit kaldığı durumla ilgilenilmiştir. Normal gerilme değerleri lifmatris ara yüzeyinde, ancak matris üzerinde hesaplanmıştır.

İçi boş liflerin yarıçaplarının değişimi ile normal gerilmeler arasındaki ilişkinin monoton olmadığı, bu gerileme değerlerinin lif kalınlığı değişimi ile ise monoton olduğu gözlemlenmiştir. Ayrıca, lifler arasındaki etkileşimin hesaplanan normal gerilme değerlerini azalttığı ortaya çıkmıştır. Eğilmenin derecesi ve elastisite modülü oranının artmasının $\sigma_{\tau \tau} / p$ değerlerini azalttığı, oysa $\sigma_{e e} / p$ değerlerini artırdığı izlenmiştir.

Uygulamada, genellikle, daha çok sayıda lifin etkileşimi söz konusudur. Dolayısıyla, bu çalışma, daha çok lifin etkileşiminin dikkate alınacağı çalışmanın temelini oluşturmaktadır. Ayrıca, bu çalışma, yapı elemanı olarak da kullanılan ve burada ele alınan kompozit malzemede normal gerilmelerin davranışı ile ilgili, uygulamaciya fikir verir.

Açıklama: Bu araştırma Yıldız Teknik Üniversitesi Bilimsel Araştırma Projeleri Koordinatörlüğü'nce desteklenmiştir. Proje No: 2014-07-03-DOP01

\section{Kaynakça}

AKBAROV, Surkay D. (2013). "Microbuckling of a DoubleWalled Carbon Nanotube Embedded in an Elastic Matrix". International Journal of Solids and Structures. 50: 25842596.
AKBAROV, Surkay D. (2012). Stability Loss and Buckling Delamination: Three-Dimensional Linearized Approach for Elastic and Viscoelastic Composites..Springer.

AKBAROV, Surkay D. KOSKER, Resat ve UCAN, Yasemen (2004). "Stress distribution in an elastic body with a periodically curved row of fibers". Mechanics of Composite Materials. 40 (3): 191-202.

AKBAROV, Surkay D. KOSKER, Resat ve UCAN, Yasemen (2006). "Stress distribution in a composite material with the row of anti-phase periodically curved fibers". International Applied Mechanics. 42 (4): 486-493.

AKBAROV, Surkay D. KOSKER, Resat ve UCAN, Yasemen (2010). "The Effect of the Geometrical Non-Linearity on the Stress Distribution in the Infinite Elastic Body with a Periodically Curved Row of Fibers". CMC:Computers, Materials, \& Continua. 17 (2): 77-102.

AKBAROV, Surkay D. KOSKER, Resat ve UCAN, Yasemen (2016). "Influence of the interaction between fibers periodically located in a composite material on the distribution of stresses in it". Mechanics of Composite Materials. 52 (2): 243-256.

AKBAROV, Surkay D. ve KOSKER, Resat (2003a). "On a stress analysis in the infinite elastic body with two neighbouring curved fibers". Composites Part B: Engineering. 34 (2): 143150 .

AKBAROV, Surkay D. ve KOSKER, Resat (2003b). "Stress Distribution Caused By Anti-Phase Periodical Curving of Two Neighbouring Fibers in a Composite Material". European Journal of Mechanics A/Solids. 22 : 243-256.

AKBAROV, Surkay D., GUZ, Aleksander N. (1985). "Method of Solving Problems in the Mechanics of Fiber Composites With Curved Structures”. Soviet Applied Mechanics. March: 777785.

AKBAROV, Surkay D., GUZ, Aleksander N. (2002). "Mechanics of curved composites (piecewise homogenous body model)". International Applied Mechanics. 38 (12): 1415-1439.

AKBAROV, Surkay D.-GUZ, Aleksander N. (2000). Mechanics of Curved Composites. Kluwer Academic Publishers.

CORTEN, H. T., BROUTMAN, L. J., \& KROCH, R. H. (1967). Modern Composite Materials. Micromechanics and Fracture Behavior of Composites. Addison-Wesley, Reading, Massachusetts.

ERDEN, S. „HO K. (2017), Fiber Technology for FiberReinforced Composites, Seydibeyoglu M O, Mohanty A K, Misra M, Editor, Woodhead Publishing.

GUZ, Aleksander N. (2003). "On one two-level model in the mesomechanics of compression fracture of Cracked Composites". International Applied Mechanics. 39 (3):274285.

GUZ, Aleksander N. and DEKRET, V.A., (2008)." On two models in the three-dimensional theory of stability of composites". International Applied Mechanics. 44 (8): 839854.

KASHTALYAN, M. Yu. (2005). "On deformation of ceramic cracked matrix cross-ply composites laminates". International Applied Mechanics. 41 (1):37-47. 
KELLY, Anthony (1998), "Composite Materials: impediments do wider use and some suggestions to overcome these", Proceeding Book ECCM-8, 3-6 June, Napoles-Italy, Vol. I, pp. 15-18.

KÖŞKER, Reşat (2002). Tek Yönlü Lifli Elastik ve Viskoelastik Kompozitlerin İç stabilitesi ve Gerilme Durumuna Ait Bazı Problemler, YTÜ, Fen Bilimleri Enstitüsü, Doktora Tezi, İstanbul.

KOSKER, Resat ve GULTEN, İsmail (2020). "Stress Distribution in Elastic Media Containing Hollow Fiber with Periodic Curvature," European Journal of Science and Technology. 19: 809-820.

KOSKER, Resat ve AKBAROV, Surkay D. (2003). "Influence of the interaction between two neighbouring periodically curved fibers on the stress distribution in a composite material" Mechanics of Composite Materials. 39 (2): 165-176.

KOSKER, Resat ve UCAN, Yasemen (2004). "On the normal stresses in the elastic body with Periodically curved row fibres "Journal of Engineering and Natural Sciences (Mühendislik ve Fen Bilimleri Dergisi)- Sigma. 4: 294-304.

MALIGINO, A.R. \& WARRIOR, N.A. \& LONG, A.C. (2009). "Effect on inter-fibre spacing on damage evolution in unidirectional (UD) fibre-reinforced composites.". European Journal of Mechanics - A/Solids., 28: 768-776.

QİAN, D.; DİCKEY, E. C.; ANDREWS, R.; RANTELL, T. (2000): "Load transfer and deformation mechanisms of carbon nanotube-plytyrene composites". Applied Physics Letters. 76 (20): 2868-2870.

ZHUK, Y.A. and GUZ, I.A. (2007). “ Features of plane wave propagation along the layers of a prestrained nanocomposite". International Applied Mechanics. 43 (4): 361-379.

WATSON, G.N. (1966), A Treatise on the Theory of Bessel Functions, Cambridge. 